\title{
消除大容量低 $\mathrm{NO}_{x}$ 切向燃煤锅炉烟温偏差 现场试验
}

\author{
刘福国 $^{1}$ 徐 伟 $^{2}$ 胡代军 ${ }^{2}$ 咸兰辉 ${ }^{2}$
}

(1. 山东电力集团公司电力科学研究院 济南 250002;

2. 大唐黄岛发电有限责任公司 青岛 266500)

\begin{abstract}
摘要: 回顾切向燃烧的技术发展, 分析最新设计的大容量切向燃烧系统在降低 $\mathrm{NO}_{x}$ 排放和控制烟温偏差方面的技术特点。在 一台 $660 \mathrm{MW}$ 锅炉上, 进行气温偏差的调整试验, 调整的主要思路是在保证恰当的空气分级、满足环保要求的 $\mathrm{NO}_{x}$ 排放的情 况下, 采用分离燃尽风的水平角度来协调消旋所需要的旋转动量矩; 定量研究燃烧器调温摆角、炉膛风箱压差、分离燃尽风 水平角度和风量以及紧凑燃尽风风量对炉膛上部的分隔屏出口气温偏差的影响, 结果表明, 该锅炉经过优化调整后, 消除近 年来一直困扰电力生产的炉膛烟温偏差问题, 并保证较低的 $\mathrm{NO}_{x}$ 排放量 $449.0 \mathrm{mg} / \mathrm{m}^{3}$ 和较高的燃烧效率; 提出在实际生产中 将该类大容量低 $\mathrm{NO}_{x}$ 切向燃煤锅炉的气温偏差投入自动控制的设想。
\end{abstract}

关键词: 煤燃烧 切向燃烧锅炉 烟温偏差 $\mathrm{NO}_{x}$ 排放 残余旋转

中图分类号: TK22

\section{Field Tests Elimination of the Gas Temperature Deviation in Large Scale, Advanced Low NO$x$, Tangentially Fired Pulverized Coal Boiler}

\author{
LIU Fuguo $^{1} \quad$ XU Wei $^{2} \quad$ HU Daijun $^{2} \quad$ XIAN Lanhui ${ }^{2}$ \\ (1. Shandong Electric Power Research Institute, Jinan 250002; \\ 2. Datang Huangdao Power Generation Co., Ltd., Qingdao 266500)
}

\begin{abstract}
The developments of coal tangentially fired technology are concisely reviewed and technical details of reducing $\mathrm{NO}_{x}$ emissions combined with alleviating the gas temperature deviation are discussed for a newly designed combustion system in pulverized coal boiler. Field tests are carried out to alleviate the steam temperature deviation in the crossover pass of a $660 \mathrm{MW}$ boilers, major attention was paid to the coordination of the rotational momentum required for the residual swirl elimination with the air staged flow necessary for reducing $\mathrm{NO}_{x}$ emissions by the changes in yaw angles of separated over-fire air. The effects of nozzle tilt angle for reheater steam temperature regulation, pressure difference of furnace with wind box, flow-rates and yaw angles of over-fire air, are quantitatively investigated on steam temperature deviation in division platen superheaters in the upper furnace, it shows that the gas temperature deviation are eliminated in tangentially fired pulverized coal boiler by field combustion optimization, and keeping low $\mathrm{NO}_{x}$ emissions of $449.0 \mathrm{mg} / \mathrm{m}^{3}$ and higher combustion efficiency in the meantime. Suggestions of putting steam temperature deviation into automatic control are proposed in large scale, advanced low $\mathrm{NO}_{x}$, tangentially fired pulverized coal boiler.
\end{abstract} Key words: Coal combustion Tangentially fired boiler Gas temperature deviation $\mathrm{NO}_{x}$ emissions Residual swirl

\section{0 前言}

煤粉切向燃烧是一种在炉膛内整体组织的燃 烧技术，具有易于引燃、混合强烈、燃尽度高、对 易结焦煤适应性强等优点, 因而得到广泛应用; 随

20121227 收到初稿, 20130516 收到修改稿
着氮氧化物排放法规的实施, 近 20 年来, 为适应环 保要求, 切向燃烧进行了重要的技术改进, 其中, 基于 $\mathrm{NO}_{x}$ 生成机理而做出的最简单有效的改进是将 部分二次风偏离一次风方向, 以较大的假想切圆射 入炉膛, 一次风被旋绕的二次风包围, 在炉膛横断 面产生燃料梯度，使着火阶段只有少量空气和煤粉 混合，形成同心燃烧系统(Concentric firing system, 
CFS), 实现水平方向分段燃烧; 另一项改进是从主 燃烧区分流出一部分二次风, 通过主燃烧器上部的 喷嘴送入炉内(Over fire air, OFA), 使主燃烧区形成 缺氧燃烧, 在上部燃尽区送入燃烧所需的剩余空气, 实现炉膛坚向空气分级, 这些改进有效地降低了 $\mathrm{NO}_{x}$ 排放量 ${ }^{[1-4]}$; 但环保需求不是切向燃烧技术改进 的唯一动力, 随着容量增加, 切向燃烧炉膛上部及 水平烟道两侧的烟温偏差随之增大, 危及受热面的 安全运行, 爆管事故时有发生 ${ }^{[5-6]}, 1994$ 年, 美国 大型锅炉制造商 ABB-CE 公司率先实施 GSEIC 计 划, 着力解决切向燃烧的烟气偏差问题, 随后, 锅 炉生产商和科研机构对切向燃烧进行技术改进, 试 图解决烟温偏差问题。

切向燃烧喷口射流的几何中心线与位于炉膛 中央的一个或多个同心水平假想圆相切, 在炉内形 成旋转上升火焰, 锅炉的烟温偏差呈现出与这种燃 烧特点相关的规律性, 当燃烧切圆逆时针旋转时, 炉膛出口水平烟道右侧的烟温高于左侧, 位于该处 的屏式再热器和对流过热器的工质温度呈右侧高左 侧低的特性, 而炉膛上部的分隔屏受热面工质温度 呈左侧高右侧低的分布 ${ }^{[6-7]}$; 当燃烧切圆顺时针旋转 时, 上述温升特性恰好相反。试验测试和数值模拟 结果表明 ${ }^{[8-10]}$, 切向燃烧气流在炉膛出口存在残余 旋转, 对于逆时针旋转的切向燃烧锅炉, 炉膛上部 左侧气流有一个速度衰减、滞止及反向加速过程, 造成较强的气流扰动, 因此, 左侧受热面在辐射换 热的基础上附加了对流换热强化效应, 且左侧烟气 往后运动的阻力也较大; 而炉膛上部右侧气流扰动 较小, 平稳加速流向炉后, 因惯性速度指向炉后,

一部分气流还直接短路流过折焰角上部, 形成 “烟 气走廊”; 这种流动模式造成炉膛上部的分隔屏受热 面吸热量呈左侧高右侧低的分布, 而进入水平烟道 的烟气流量呈右侧多左侧少的分布, 这是造成烟温 和气温偏差的根本原因 ${ }^{[10-11]}$ 。

消除或控制炉膛出口气流的残余旋转是解决 烟温偏差的基本措施，至今已有多种反切射流消旋 设计, 取得了不同效果。燃烧器喷口射流的动量和 角度决定了炉内气流旋转强度, CFS 同心燃烧系统 的偏转二次风假想切圆增大, 增加了气流旋转强度, 对降低烟温偏差是不利的, 这种低 $\mathrm{NO}_{x}$ 切向燃烧系 统在随后改进设计时, 采用了二次风与一次风大夹 角 $\left(17^{\circ} \sim 25^{\circ}\right)$ 反切布置方式, 以降低气流旋转强度, 实践证明, 这种设计在高负荷时烟温偏差有所降低, 但低负荷时主燃烧区气流旋转强度过小，甚至会变 为反向旋转, 形成不稳定燃烧区 ${ }^{[6,12]}$ 。在切向燃烧 锅炉上实施的其他消旋方案还有: (1) 一次风煤粉气
流改为反切, 但采用一、二次风微量偏转(夹角在 $5^{\circ}$ 左右)的布置方式 ${ }^{[13-14]}$; (2) 对于配有中间储仓式制 粉系统的锅炉, 进行顶部三次风的反切改造 ${ }^{[15-17]}$;

(3) 将低 $\mathrm{NO}_{x}$ 燃烧系统的 OFA 或一部分上层二次风 改为反切 ${ }^{[18-20]}$ 。这些方案对降低烟温偏差有一定效 果, 但常常会引起炉内混合减弱、飞灰可燃物升高、 煤粉刷墙结渣、低负荷稳燃或降低 $\mathrm{NO}_{x}$ 排放的能力 受到影响等副作用 ${ }^{[6,15,19]}$ 。

因此, 切向燃烧喷口射流的偏转、反切和坚向 布置是降低 $\mathrm{NO}_{x}$ 排放和减少烟温偏差的主要手段, 喷口射流的角度、流量、动量和位置是关键设计参 数, 美国 ABB-CE 公司在低 $\mathrm{NO}_{x}$ 同心燃烧系统(Low $\mathrm{NO}_{x}$ concen-tric firing system, LNCFS)开发过程中, 坚向空气分级经过紧凑燃尽风(Closed-coupled OFA, CCOFA)、分离燃尽风(Separated OFA, SOFA)以及紧 凑燃尽风和分离燃尽风相结合等多次尝试, 才最终 确定了合理的燃尽风风量和喷口位置 ${ }^{[4,18]}$; 近年来, 切向燃烧技术的发展就是采用试验分析和数值模拟 手段对上述关键参数以及它们的组合方式进行优 化 ${ }^{[21-23]}$, 使这些参数在降低 $\mathrm{NO}_{x}$ 排放和减少烟温偏 差方面达到良好的协调, 同时, 在锅炉负荷变化时, 保证主燃烧区气流旋转状态稳定，保持该燃烧技术 特有的易于引燃、混合强烈的优势。

分析近年投产的一台大容量超临界压力直流 锅炉低 $\mathrm{NO}_{x}$ 切向燃烧系统的设计特点, 并采用现场 试验研究了最新设计的该类燃烧系统在降低烟温偏 差方面所能达到的效果, 文献[24-26]多采用相似模 型或数值模拟方法研究烟温偏差问题, 还鲜见通过 锅炉的调整试验消除烟温偏差的详细报道。

\section{1 超临界压力锅炉低 $\mathrm{NO}_{x}$ 燃烧系统}

某电厂 6\#机组 2007 年投产, 锅炉是上海锅炉 厂生产的 SG2102/25.40-M953 型超临界参数、一次 中间再热直流锅炉, 采用单炉膛切向燃烧、变压运 行、露天悬吊、固态排渣, 再热汽采用燃烧器摆动 调温, 二次风喷嘴的摆动范围可达 $\pm 30^{\circ}$, 煤粉喷嘴 的摆动范围为 $\pm 20^{\circ}$; 在燃烧器 $A B 、 C D 、 E F$ 二次风 室中配置了三层共 12 支轻油枪; 设计燃用充州烟 煤, 配置 6 台 ZGM113G 型中速辊式磨煤机, 每台 磨煤机供一层 4 只燃烧器, 因此, 共设有 $A 、 B 、 C 、$ $D 、 E 、 F$ 共 6 层煤粉喷嘴, 煤粉喷嘴四周布置有燃 料风(周界风); 在每相邻 2 层煤粉喷嘴之间布置有 1 层辅助风喷嘴; 在主风箱下部设有 1 层火下风 (Underfire air, UFA)喷嘴; 主风箱上部设有 2 层紧凑 燃尽风和 5 层分离燃尽风; 各种风的设计风温、风 
速和风率见表 1 , 这些风组成了低 $\mathrm{NO}_{x}$ 同轴燃烧系 统, 燃烧器的详细布置布置见图 1、2。

表 1 设计风速、风温和风率

\begin{tabular}{|c|c|c|c|}
\hline 序号 & \multicolumn{2}{|c|}{ 名称 } & 数据 \\
\hline 1 & \multicolumn{2}{|c|}{ 二次风速度/(m/s) } & 57.0 \\
\hline 2 & \multicolumn{2}{|c|}{ 二次风温度 $/{ }^{\circ} \mathrm{C}$} & 335 \\
\hline \multirow{4}{*}{3} & \multirow{4}{*}{ 二次风率(\%) } & SOFA & 30.0 \\
\hline & & CCOFA & 10.0 \\
\hline & & 周界风 & 10.0 \\
\hline & & 辅助风 & 28.09 \\
\hline 4 & \multicolumn{2}{|c|}{ 一次风喷口速度 $/(\mathrm{m} / \mathrm{s})$} & 25 \\
\hline 5 & \multicolumn{2}{|c|}{ 一次风温度 ${ }^{\circ} \mathrm{C}$} & 75 \\
\hline 6 & \multicolumn{2}{|c|}{ 一次风率(\%) } & 16.91 \\
\hline
\end{tabular}

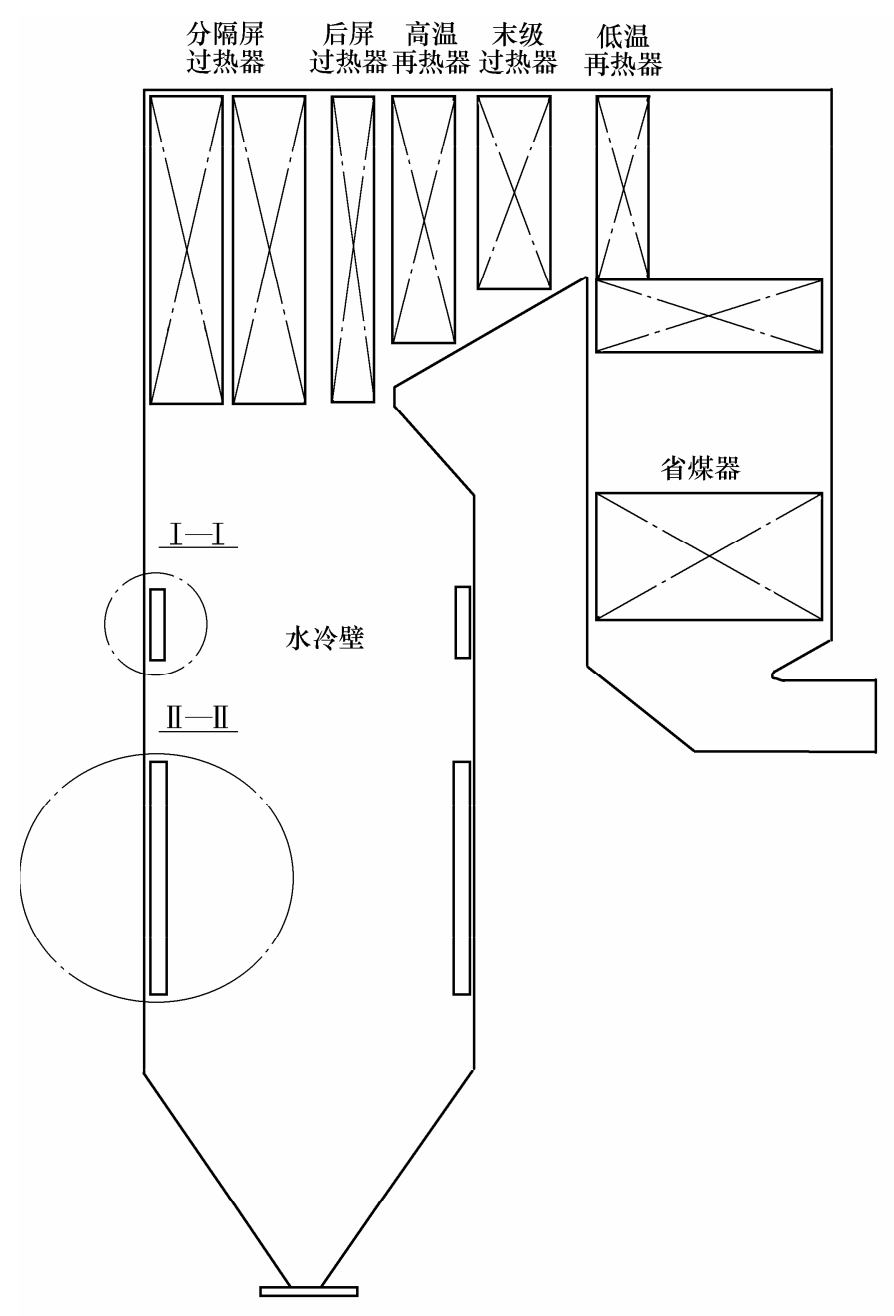

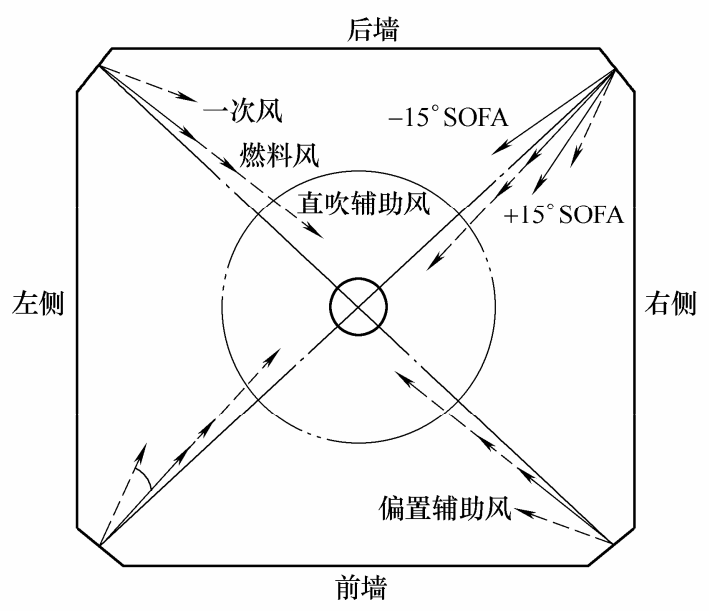

图 1 低 $\mathrm{NO}_{x}$ 同轴燃烧系统

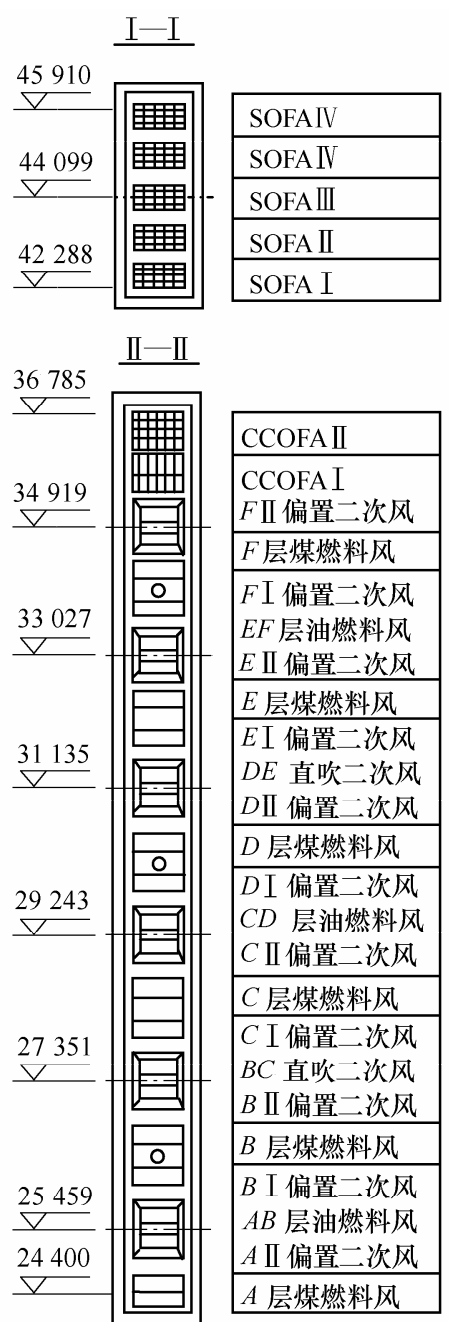

图 2 二次风喷口坚向布置及炉内受热面

该锅炉低 $\mathrm{NO}_{x}$ 同心燃烧系统(LNCFS)的设计特 点如下。

(1) 通过在炉膛的不同高度布置紧凑燃尽风和 分离燃尽风, 将炉膛分成三个相对独立的部分：初 始燃烧区、 $\mathrm{NO}_{x}$ 还原区和燃料燃尽区。每个区域的 过量空气系数由三个因素控制: (1) 总的 OFA 风量;
(2) CCOFA 和 SOFA 的风量分配; (3) 总过量空气系 数。这种改进的空气分级方法通过优化每个区域的 过量空气系数, 在有效降低 $\mathrm{NO}_{x}$ 排放的同时, 最大 限度地提高燃烧效率。

(2) 采用多隔仓辅助风设计的同心切圆燃烧方 式。相邻煤粉喷嘴之间的辅助风喷嘴包括 1 只直吹 
风喷嘴和 2 只 CFS 偏置风喷嘴; 直吹辅助风提供油 枪或煤粉着火需要的空气, 偏置辅助风气流在水平 方向分级, 在初始燃烧阶段推迟了空气和煤粉的混 合, 减少 $\mathrm{NO}_{x}$ 生成; 由于一次风煤粉气流被偏转的 二次风气流裹在炉膛中央, 形成富燃料区, 在燃烧 区域及上部水冷壁附近则形成富空气区，能有效防 止炉内结渣和高温腐蚀。

(3) 在控制炉膛出口烟温偏差方面进行了总体 设计, 采用反切分离燃尽风在燃尽区气流进行消旋, SOFA 可水平摆动, 摆角可水平调整 $+15^{\circ}$ 到 $-15^{\circ}$, 图 1 表示了可水平调整摆角的喷嘴设计, 这种设计在 相同的流量下可获得不同的消旋动量矩, 同时还能 加强燃尽区的扰动和混合, 降低一氧化碳体积分数 和飞灰含碳量。分离燃尽风风量和角度在现场通过 燃烧优化确定。

分离燃尽风水平角度调整是一种独特的设计, 能够对低 $\mathrm{NO}_{x}$ 排放所需的分级风量和消除烟温偏差 所需的反切风量进行协调, 合理的分离燃尽风水平 反切角度既能保证适度的空气分级, 又满足消旋需 要的反切风风量。

图 2 所示的每角主燃烧器配有 26 只风门挡板, 每层煤粉风室上下的两只偏置辅助风挡板由一套执 行机构控制, 每角总计有 20 只气动执行机构; 每 角 SOFA 燃烧器配有 5 只风门挡板, 相应配有 5 只 执行机构。

\section{3 消除烟温偏差的现场试验}

\section{1 受热面布置}

锅炉给水经省煤器进入水冷壁, 水在水冷壁内 吸热后引入汽水分离器, 分离出的蒸汽经炉顶过热 器流入后烟井包覆过热器, 再分别流经分隔屏、后 屏和末级过热器, 进入汽机高压缸, 上述各受热面 在炉膛内的位置见图 2; 在分隔屏过热器和后屏过 热器之间设有一级喷水减温器, 在后屏过热器和末 级过热器之间设有二级喷水减温器, 过热器系统布 置见图 3 。

\section{2 烟温和气温偏差}

未进行调整之前, 炉膛顶部及水平烟道存在着 显著的烟温和气温偏差。如图 1 所示, 由于炉内燃 烧切圆是顺时针旋转, 右侧分隔屏的出口蒸汽温度 明显高于左侧, 而末级过热器的吸热量呈现左侧多 右侧少的分布, 如图 3 所示, 该锅炉在后屏和末过 之间采用交叉混合设计, 对于分隔屏和末级过热器

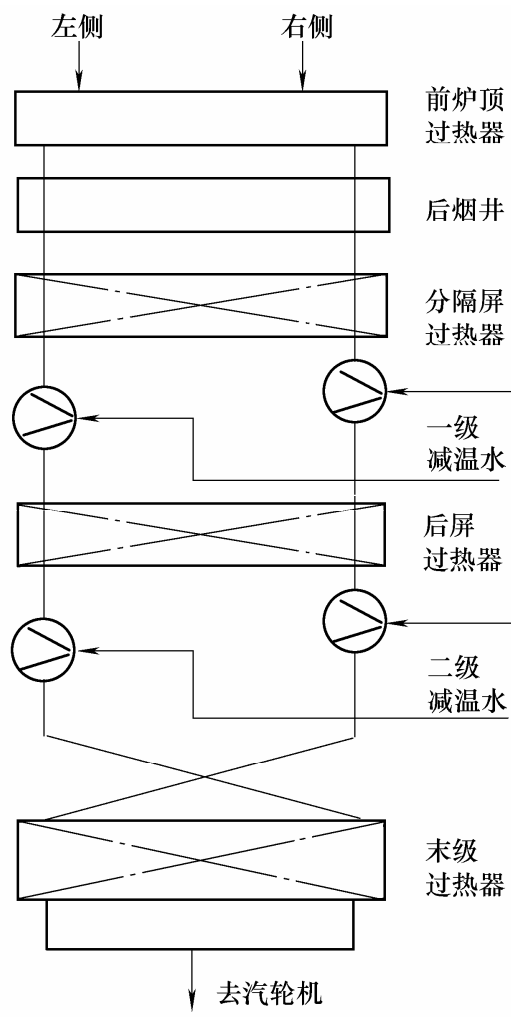

图 3 过热汽系统

的左右侧气温的分布特性，这种设计会造成温度偏 差叠加, 是不合理的 ${ }^{[27]}$ 。

该锅炉在运行中, 分隔屏出口蒸气温度偏差是 急迫解决的问题, 高负荷时右侧气温比左侧高 20 ${ }^{\circ} \mathrm{C}$ 以上, 见图 4, 导致右侧一级减温水开度为 $100 \%$, 减温水量达到 $50 \mathrm{t} / \mathrm{h}$, 而左侧减温水经常处于关闭 状态; 采用二级喷水减温将末级过热器入口左、右 两侧气温调节到相同温度的情况下, 在末级过热器 出口, 左侧气温明显高于右侧, 见图 5 ; 这是切向 燃烧锅炉典型的气温分布型式。

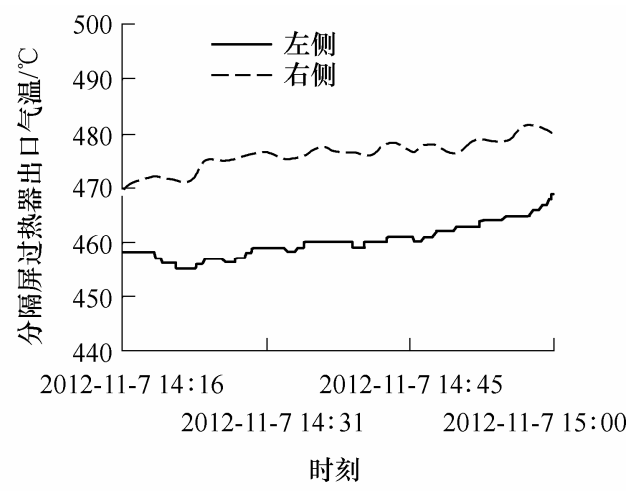

图 4 分隔屏出口左右两侧气温

\section{3 降低气温和烟温偏差试验}

在进行气温和烟温偏差调整之前, 对 5 层分离 燃尽风的水平角度进行了调整和定位, 图 2 中的III、 


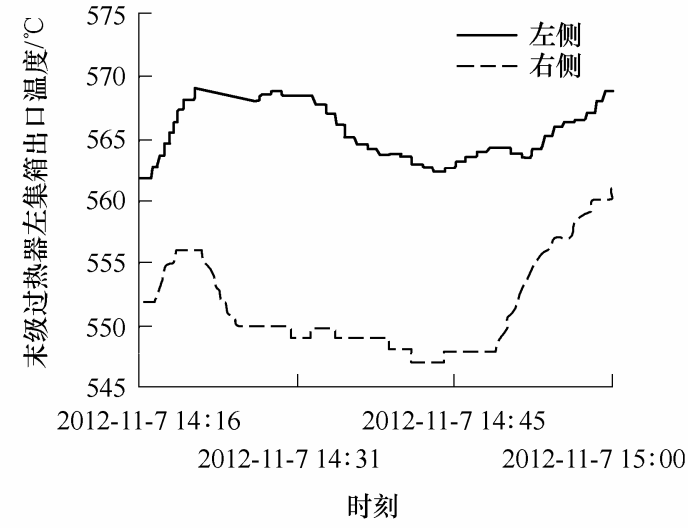

图 5 末级过热器出口左右两侧气温

IV、V层 SOFA 风的水平角度与一次风反切 $10^{\circ}$, II 层 SOFA 风与一次风水平角度相同, I 层 SOFA 风与一次风顺切 $15^{\circ}$, 试验证明, 这种角度配合能 够对分级风量和反切风量进行良好的协调, 同时保 证燃尽区气流混合强烈, 利于一氧化碳和飞灰燃尽。 气温和烟温偏差试验分 4 个阶段共进行 12 个项目的
调整, 每次调整只进行单因素的变化, 这些调整项 目和顺序见表 2 和图 6。如图 6 所示，2012 年 11 月 7 日 13:29, 机组带 $550 \mathrm{MW}$ 负荷, 右、左分隔 屏出口气温偏差接近 $20{ }^{\circ} \mathrm{C}$, 这是平时普遍的运行 状态。

表 2 气温和烟温偏差调整项目

\begin{tabular}{cccc}
\hline 代号 & 调整项目 & $\begin{array}{c}\text { 开始 } \\
\text { 时间 }\end{array}$ & $\begin{array}{c}\text { 结束 } \\
\text { 时间 }\end{array}$ \\
\hline (1) & 炉膛风箱压差由 $0.465 \mathrm{kPa}$ 降低到 $0.335 \mathrm{kPa}$ & $13: 32$ & $13: 59$ \\
(2) & 再热汽调温摆角由 $91 \%$ 降低到 $50 \%$ & $13: 36$ & $13: 58$ \\
(3) & 炉膛风箱压差由 $0.335 \mathrm{kPa}$ 升高到 $0.485 \mathrm{kPa}$ & $14: 01$ & $14: 19$ \\
(4) & SOFA-V 开度由 79\%降低为 $0 \%$ & $14: 15$ & $14: 27$ \\
(5) & CCOFA I、CCOFA II 开度由 76\%升高 $100 \%$ & $14: 15$ & $14: 24$ \\
(6) & SOFA-IV 开度由 76\%降低到 54\% & $14: 27$ & $14: 32$ \\
(7) & SOFA-I 开度由 100\%降低到 50\% & $14: 45$ & $14: 56$ \\
(8) & SOFA-V 开度由 $0 \%$ 升高到 $100 \%$ & $14: 46$ & $14: 51$ \\
(9) & SOFA-II 开度由 95\%降低到 46\% & $14: 49$ & $14: 58$ \\
(10) & SOFA-IV 开度由 54\%升高到 $100 \%$ & $14: 52$ & $14: 59$ \\
(11) & SOFA-III 开度由 50\%升高到 $100 \%$ & $14: 58$ & $15: 00$ \\
(12) & SOFA-I 开度由 50\%降低到 $20 \%$ & $15: 09$ & $15: 22$ \\
\hline
\end{tabular}

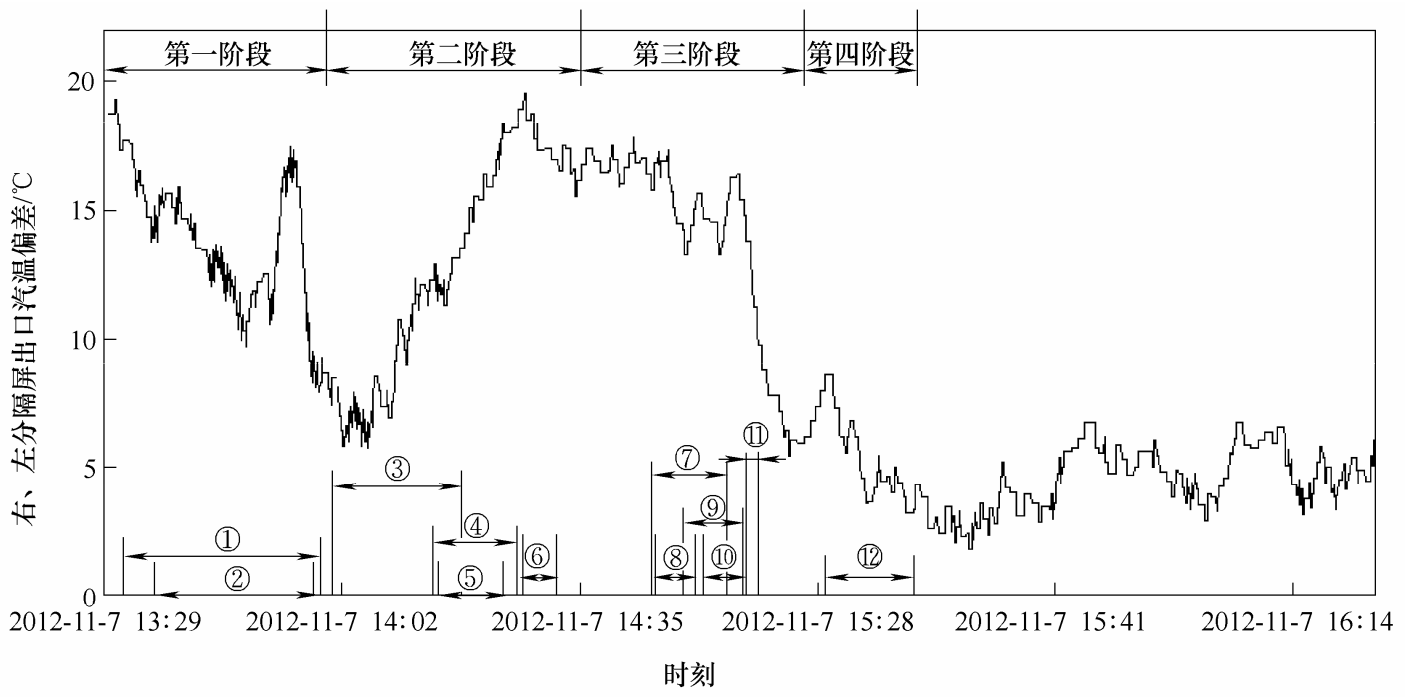

图 6 分隔屏出口气温偏差变化

试验过程先考察了不同变量变化对气温偏差 的影响, 最后通过对关键变量的调整, 将分隔屏出 口两侧气温偏差降低到约 $4{ }^{\circ} \mathrm{C}$, 左、右侧一级减温 水流量分别是 $0 \mathrm{t} / \mathrm{h}$ 和 $4 \mathrm{t} / \mathrm{h}$; 在末级过热器出口, 基 本消除了气温偏差; 调整过程中, 分隔屏出口右、 左两侧气温偏差变化见图 6。从表 2 和图 6 可以看 出如下。

(1) 第一阶段包括(1)、(2)两个调整项目，燃烧 器摆角和炉膛-风箱压差的降低对减少分隔屏出口 气温偏差有明显好处; 但炉膛-风箱压差降低意味着 炉膛进风量减少，导致未燃尽损失增加。

(2) 第二阶段包括(3)、(4)、(5)、(6)四个调整项
目, 首先将炉膛风箱压差升高到合理的数值, 这进 一步验证了气温偏差与炉膛-风箱压差的关系; 随 后, 将 SOFA-V 的开度由 79\%降低到 $0 \%$ 、SOFA-IV 开度由 76\%降低到 54\%、CCOFA I 和 CCOFA II 开 度由 $76 \%$ 升高 $100 \%$, 由于 SOFA-V、SOFA-IV 水 平角度与一次风反切 $10^{\circ}$, CCOFA I 和 CCOFA II 的 角度与一次风相同, 这些调整导致消旋风反切动量 矩减少, 气温偏差升高到调整开始前的数值。

(3) 第三阶段包括(7)、8、(9)、(10、(11)五个调 整项目, 将反切 $10^{\circ}$ 的III、IV、V层 SOFA 风门的 开度提高到 $100 \%$, 减少 I 、 II 层 SOFA 风门的开 度, 它们与一次风顺切或角度相同, 这时, 气温偏 
差有显著降低。

第二阶段和第三阶段从不同的方向验证了 SOFA 风对气温偏差的影响。

(4) 第四阶段包括12 一个调整项目, 进一步关 小与一次风顺切 $15^{\circ}$ 的 I 层 SOFA 风门的开度, 气 温偏差有一定降低。

\section{4 锅炉效率和 $\mathrm{NO}_{x}$ 排放测试}

经过现场调整, 该大容量低 $\mathrm{NO}_{x}$ 切向燃煤锅炉 的气温偏差得到消除, 现场测试表明, $\mathrm{NO}_{x}$ 排放量 达到 $449.0 \mathrm{mg} / \mathrm{m}^{3}$, 并保持了良好燃尽性能, 锅炉效 率达到 $94.835 \%$ 。

\section{4 结论}

(1) 切向燃烧锅炉炉膛出口烟温和气温偏差由 气流残余旋转引起, 近年来进行的反切气流消旋改 造对降低烟温和气温偏差有一定效果, 但时常引起 飞灰可燃物升高、煤粉刷墙结渣、低负荷稳燃能力 受到影响等副作用, 且没有完全解决烟温偏差问题。

(2) 随着大容量低 $\mathrm{NO}_{x}$ 切向燃烧系统的技术改 进, 最新设计的该类燃烧系统在降低 $\mathrm{NO}_{x}$ 排放和消 除烟温偏差方面取得了良好的效果; 紧凑燃尽风、 水平角度可调的分离燃尽风与多隔仓辅助风的同心 切圆燃烧系统相结合, 能够对分级风量和消旋反切 风量进行有效协调, 在满足 $\mathrm{NO}_{x}$ 排放的情况下, 有 效控制炉膛出口的气温偏差, 并保持切向燃烧原有 的优越性。

(3) 现场优化调整是发挥大容量低 $\mathrm{NO}_{x}$ 切向燃 烧系统设计性能的必要途径, 试验表明, 燃烧器调 温摆角、炉膛风箱压差、分离燃尽风水平角度和开 度以及紧凑燃尽风开度是降低气温偏差的重要手 段; 建议进一步探索气温偏差的影响因素和规律, 在实际生产中将气温偏差投入自动控制。

\section{参 考 文 献}

[1] LUIS I D, CRISTOBAL C, JAVIER P. Numerical investigation of $\mathrm{NO}_{x}$ emissions from a tangentially-fired utility boiler under conventional and overfire air operation[J]. Fuel, 2007, 87 (7): 1259-1269.

[2] CHOENG R C, CHANG N K. Numerical investigation on the flow, combustion and $\mathrm{NO}_{x}$ emission characteristics in a $500 \mathrm{MW}$ tangentially fired pulverized-coal boiler[J]. Fuel, 2009, 88 (9): 1720-1731.

[3] 胡志宏, 郝卫东, 薛美盛, 等. $1000 \mathrm{MW}$ 超超临界燃 煤锅炉燃烧与 $\mathrm{NO}_{x}$ 排放特性试验研究 $[\mathrm{J}]$. 机械工程学
报，2010，46(4): 105-109.

HU Zhihong, HAO Weidong, XUE Meisheng, et al. Experimental study on combustion and $\mathrm{NO}_{x}$ emission characteristic of $1000 \mathrm{MW}$ ultra-supercritical coal-fired boiler[J]. Journal of Mechanical Engineering, 2010, 46(4): 105-109.

[4] 黄少鸪. 美国切圆火炬燃烧锅炉运用集中燃烧技术治 理 $\mathrm{NO}_{x}$ 排放 $[\mathrm{J}]$. 广东电力, $2003,16(2)$ : 1-4.

HUANG Shaoe. Application of concentric firing technology in controlling $\mathrm{NO}_{x}$ emission of tangential firing boilers in America [J]. Guangdong Electric Power, 2003, 16(2): 1-4.

[5] XU Minghou, YUAN Jianwei, DING Shifa, et al. Simulation of the gas temperature deviation in large-scale tangential coal fired utility boilers[J]. Computer Methods in Applied Mechanics and Engineering, 1998, 155 (3-4): 369-380.

[6] 姜义道, 李永兴, 刘明仁. 四角切圆燃烧大容量电站锅 炉烟气参数场偏置问题的研究 [J]. 动力工程, 1994, 14(5): 16-21.

JIANG Yidao, LI Yongxing, LIU Mingren. The deviation problem of flue gas parameter field in four corner trangentially fired utility boilers[J]. Power Engineering, 1994, 14 (5): 16-21.

[7] 赵晴川, 吴晓武, 陈方高, 等. 2102t/h 超临界锅炉气温 偏差试验研究及分析 $[\mathrm{J}]$. 电站系统工程, 2008, 24(2): 17-20.

ZHAO Qingchuan, WU Xiaowu, CHEN Fanggao, et al. Steam temperature deviation test and analysis for $2102 \mathrm{t} / \mathrm{h}$ supercritical boiler[J]. Power System Engineering, 2008, 24 (2): 17-20.

[8] ZHOU Yuegui, XU Tongmo, HUI Shien, et al. Experimental and numerical study on the flow fields in upper furnace for large scale tangentially fired boilers[J]. Applied Thermal Engineering, 2009, 29 (4): 732-739.

[9] XU Lijun, JAMIL A K, CHEN Zhihang. Thermal load deviation model for superheater and reheater of a utility of a utility boiler[J]. Applied Thermal Engineering, 2000, 20(6): 545-558.

[10] HO Y P, SE H B, YOUNG J K, et al. Numerical and experimental investigations on the gas temperature deviation in a large scale, advanced low $\mathrm{NO}_{x}$, tangentially 
fired pulverized coal boiler[J]. Fuel, 2013, 104(2): 641-646.

[11] CHUNGEN Y, LASSE R, THOMAS J C. Further study of the gas temperature deviation in large-scale tangentially coal-fired boilers[J]. Fuel, 2003, 82(9): 1127-1137.

[12] 宋超, 马喜晨, 朱娅. 切向燃烧锅炉气流反旋现象试验 研究[J]. 黑龙江电力技术，1999，21(5)：58-61.

SONG Chao, MA Xichen, ZHU Tong. Experimental study on reverse rotation in a tangentially firing boiler[J]. Heilongjiang Electric Power, 1999, 21(5): 58-61.

[13] 赵宗让, 章明川. 切向燃烧锅炉一次风逆向偏转燃烧技 术的应用研究 $[\mathrm{J}]$. 中国电机工程学报, 1997, 17(1): 54-57.

ZHAO Zongrang, ZHANG Mingchuan. Application study on the combustion technology by counter-offsetting primary air and coal steam for tangentially fired boilers[J]. Proceeding of CSEE, 1997, 17(1): 54-57.

[14] 赵宗让, 章明川. 四角切向燃烧锅炉煤粉射流逆向稳燃 技术的研究开发 [J]. 燃烧科学与技术, 1997, 3(1): 88-96.

ZHAO Zongrang, ZHANG Mingchuan. Research and development on stabilizing pulverized coal combustion technology by counter-offsetting primary air and coal streams for $\mathrm{t}$-fired boilers[J]. Journal of Combustion Science and Technology, 1997, 3(1): 88-96.

[15] 潘朝红, 梁化忠, 张新亮, 等. 反切风技术减轻烟温偏 差试验研究 $[J]$. 河北电力技术, 2002，21(5)：42-45.

PAN Zhaohong, LIANG Huazhong, ZHANG Xinliang, et al. An experimental research on reducing flue gas temperature deviation by counter-tangential air technology[J]. Hebei Electric Power, 2002, 21(5): 42-45.

[16] 吴风廷, 鲁学农, 曾汉才, 等. 大型锅炉水平烟道左右 两侧烟温偏差的研究 [J]. 燃烧科学与技术, 1998, 4(3): 258-262.

WU Fengting, LU Xuenong, ZENG Hancai, et al. Experimental research on gas side temperature imbalance in horizontal pass of large boiler[J]. Journal of Combustion Science and Technology, 1998, 4(3) : 258-262.

[17] 段学农, 龚柏云, 李文军, 等. 三次风反切消除炉膛出 口烟温偏差试验研究 [J]. 湖南电力, 2000, 20(1): 3-8. DUAN Xuenong, GONG Boyun, LI Wenjun, et al. An experimental research on reducing flue gas temperature deviation by counter-tangential tertiary $\operatorname{air}[\mathrm{J}]$. Hunan Electric Power, 2000, 20(1): 3-8.

[18] 张维侠, 张建文. LNCFS 燃烧系统在 600MW 超临界机 组中的应用 [J]. 锅炉技术，2007，38(6): 42-45.

ZHANG Weixia, ZHANG Jianwen. The application of LNCFS firing system in $600 \mathrm{MW}$ supercritical power plant units[J]. Boiler Technology, 2007, 38(6): 42-45.

[19] 徐秀清, 曾瑞良. 大容量锅炉四角切圆燃烧时的烟温偏 差问题 $[\mathrm{J}]$. 电站系统工程，2001，17(1): 45-50.

XU Xiuqing, ZENG Ruiliang. Discussion on gas temperature deviation of large boiler with tangential firing [J]. Power System Engineering, 2001, 17(1): 45-50.

[20] 袁益超，刘聿拯，陈之航. 大型电站锅炉烟温偏差与气 温偏差研究[J].锅炉技术，2003，34(3): 15-20.

YUAN Yichao, LIU Yuzheng, CHEN Zhihang. A study on temperature deviation of flue gas and steam in large capacity utility boilers[J]. Boiler Technology, 2003, 34(3): $15-20$

[21] 张戟, 吕俊复, 岳光溪, 等. 2008t/h 四角燃烧煤粉锅炉 烟温偏差实炉测试分析 [J]. 锅炉技术，2004，35(6): $1-5$.

ZHANG Ji, LÜ Junfu, YUE Guangxi, et al. Field measurement of gas temperature deviation in a $2008 \mathrm{t} / \mathrm{h}$ pulverized combustion boiler with tangential firing[J]. Boiler Technology, 2004, 35(6): 1-5.

[22] 周俊虎, 宋国良, 陈寅彪, 等. 2008t/h 四角切圆燃烧锅 炉炉膛出口烟温偏差的试验研究 [J]. 热力发电, 2003, 32(6): $31-35$

ZHOU Junhu, SONG Guoliang, CHEN Yinbiao, et al. Test and research of flue gas temperature deviation at the furnace exit of a 2008t/ h boiler with tangential firing $[\mathrm{J}]$. Thermal Power Generation, 2003, 32(6): 31-35.

[23] 庄恩如, 张敢, 陈朝晖, 等. 1025t/h 锅炉炉内烟气温度 分布试验研究 [J]. 锅炉技术, 1997，28(7): 5-10.

ZHUANG Enru, ZHANG Gan, CHEN Zhaohui, et al. Research on flue gas temperature distribution in 1025 t/h boiler[J]. Boiler Technology, 1997, 28(7): 5-10.

[24] FAN Weidong, LIN Zhengchun, LI Youyi, et al. Experimental flow field characteristics of OFA for large-angle counter flow of fuel-rich jet combustion technology[J]. Applied Energy, 2010, 87 (8): 2737-2745.

[25] YIN Chungen, CAILLAT S, HARION J L, et al. 
Investigation of the flow, combustion, heat-transfer and emissions from a 609 MW utility tangentially fired pulverized-coal boiler[J]. Fuel, 2002, 81(8): 997-1006.

[26] 袁益超, 刘聿拯, 陈之航. 大型电站锅炉烟温与气温分 布理论分析与试验研究[J]. 中国电机工程学报, 2002, 22(12): $56-61$.

YUAN Yichao, LIU Yuzheng, CHEN Zhihang. Theoretical analysis and experimental research on temperature distribution of flue gas and steam in large capacity utility boilers[J]. Proceeding of CSEE, 2002, 22(12): $56-61$.
[27] 姜义道, 姜祖光, 包建锋, 等. 消除国产 $600 \mathrm{MW}$ 机组 锅炉气温偏差的有效措施 [J]. 锅炉制造, 2000, 11(4): 18-21.

JIANG Yidao, JIANG Zuguang, BAO Jianfeng, et al. Effective measure for elimnating homemade $600 \mathrm{MW}$ boiler steam temperature deviation[J]. Boiler Manufacturing, 2000, 11(4): 18-21.

作者简介：刘福国(通信作者), 男, 1969 年出生, 高级工程师。主要研 究方向为锅炉性能优化。

E-mail: lephico@163.com

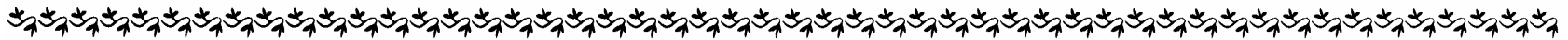

(上接第 158 页)

on porous micro heat sink for thermal management of high power LED[J]. Journal of Mechanical Engineering, 2010, 46(8): 109-113.

[7] 王辉, 汤勇, 余建军. 相变传热微通道技术的研究进展 [J]. 机械工程学报, 2010, 46(24): 101-106.

WANG Hui, TANG Yong, YU Jianjun. Recent advances of the phase change micro-channel cooling structure[J]. Journal of Mechanical Engineering, 2010, 46(24) : 101-106.

[8] SPARROW E M, RAMSEY J W, ALTEMANI C A C. Experiments on in line pin fin arrays and performance comparisons with staggered arrays[J]. ASME J. Heat Transfer, 1984, 106: 260-267.

[9] CHANG S W, YANG T L, HUANG C C, et al. Heat transfer and pressure drop in rectangular channels with attached and detached circular pin-fin array[J]. Int. J. Heat and Mass Transfer, 2008, 51: 5247-5259.

[10] MONTELPARE S, RICCI R. An experimental method for evaluating the heat transfer coefficient of liquid-cooled short pin fins using infrared thermography[J]. Experimental Thermal and Fluid Science, 2004, 28: 815-824.

[11] SAHITI N, LEMOUEDDA A, SROJKOVIC D, et al. Performance comparison of pin fin in-duct flow arrays with various pin cross-sections[J]. Applied Thermal Engineering, 2006, 26: 1176-1192.

[12] 云和明, 程林, 陈宝明, 等. 电子元件散热的优化分析 [J]. 工程热物理学报, 2006, 27(3): 496-498.

YUN Heming, CHENG Lin, CHEN Baoming, et al. Optimize analysis on the heat dissipation of electronic elements[J]. Journal of Engineering Thermophysics, 2006, 27(3): 496-498.

作者简介: 王婷(通信作者), 女, 1986 年出生, 博士研究生。主要研究 方向为车辆热管理。

E-mail: tingwang@sjtu.edu.cn

谷波, 男, 1962 年出生, 教授, 博士研究生导师。主要研究方向为制冷 系统数字化设计与车辆热管理。

E-mail: gubo@sjtu.edu.cn 